\title{
Psychedelic medicine: a re-emerging therapeutic paradigm
}

\author{
Kenneth W. Tupper PhD, Evan Wood MD PhD, Richard Yensen PhD, Matthew W. Johnson PhD
}

CMAJ Podcasts: author interview at https://soundcloud.com/cmajpodcasts/141124-ana

Competing interests: Richard Yensen is an investigator with the Multidisciplinary

Association for Psychedelic

Studies - Canada. No other competing interests were declared.

This article has been peer reviewed.

Correspondence to: Evan Wood, uhri-ew @cfenet.ubc.ca

CMAJ 2015. DOI:10.1503 /cmaj.141124
I $\mathrm{n}$ clinical research settings around the world, renewed investigations are taking place on the use of psychedelic substances for treating illnesses such as addiction, depression, anxiety and posttraumatic stress disorder (PTSD). Since the termination of a period of research from the 1950 s to the early 1970 s, most psychedelic substances have been classified as "drugs of abuse" with no recognized medical value. However, controlled clinical studies have recently been conducted to assess the basic psychopharmacological properties and therapeutic efficacy of these drugs as adjuncts to existing psychotherapeutic approaches. Central to this revival is the re-emergence of a paradigm that acknowledges the importance of set (i.e., psychological expectations), setting (i.e., physical environment) and the therapeutic clinician-patient relationship as critical elements for facilitating healing experiences and realizing positive outcomes. ${ }^{1,2}$

The public is often well-versed in the potential harms of psychedelic drugs, but much of this knowledge is from cases involving patients who used illicit substances in unsupervised nonmedical contexts. We discuss the emerging research for therapeutic purposes involving human subjects, considering both the possible benefits and the potential harms of using psychedelic agents as adjuncts to psychotherapy or counselling for mental illness.

\section{Types of psychedelic drugs}

"Psychedelic" drugs include a range of substances with varying pharmacological profiles that all have strong effects on conscious experience (Table 1). ${ }^{3-18}$ We will focus on two classes of psychedelics: classic psychedelics and "entactogens."

The classic psychedelics exert primary activity as agonists at the 5- $\mathrm{HT}_{2 \mathrm{~A}}$ receptor (e.g., lysergic acid diethylamide [LSD], psilocybin, dimethyltryptamine [DMT] and mescaline).$^{19}$ Many of these substances are found - or are close analogues of chemicals found - in plants or fungi used traditionally for millennia in spiritual or folk healing rituals, such as the ergot fungus (Claviceps purpurea) from Eurasia, morning glory (Turbina corymbosa) and peyote cactus (Lophophora williamsii) from Central and North America, and the ayahuasca brew (Banisteriopsis caapi and Psychotria viridis) from the Amazon. ${ }^{20}$

The second class of psychedelic substances, the entactogens, includes methylenedioxymethamphetamine (MDMA), which acts primarily as a serotonin-releasing agent and has effects that somewhat overlap but are substantially distinct from classic psychedelics. ${ }^{21}$

Other substances that are sometimes classified as "psychedelic" — such as ketamine (a dissociative anesthetic), scopolamine (an anticholinergic) or ibogaine (a substance with a complex neuropharmacology) - are beyond the scope of this review. This article will focus on clinically relevant studies with patient populations in which psychedelic drugs are used as adjuncts to psychotherapy. Besides a few brief mentions, we do not cover findings from research on healthy participants, although such studies have been the basis of renewed neuropharmacologic science in this field.

\section{Contexts and indications}

Some of the mental disorders for which psychedelic-assisted treatments are currently being researched include anxiety, addiction and PTSD. The findings presented in this analysis are preliminary, and most are results from small-scale pilot studies with relatively few participants. Further study is warranted before any unambiguous clinical utility may be confirmed, but the new generation of investigators is attempting to over- 
come some of the methodological weaknesses of earlier research on these substances.

\section{Anxiety}

In 2014, a small randomized controlled trial in Switzerland suggested LSD-assisted psychotherapy had the potential to reduce the anxiety associated with terminal illness. ${ }^{4}$ Twelve participants with life-threatening illness were enrolled in the study to receive treatment that involved drug-free psychotherapy sessions supplemented with two LSD-assisted sessions two to three weeks apart. The participants were randomly assigned to either the treatment group (receiving $200 \mu \mathrm{g} \operatorname{LSD}[n=8]$ ) or the active control group $(20 \mu \mathrm{g} \operatorname{LSD}[n=4]$, with an open-label crossover to $200 \mu \mathrm{g}$ LSD after the initial blinding was unmasked). At two months' follow-up, the State-Trait Anxiety Inventory (STAI) showed nonsignificant reductions in

Table 1: Psychedelic agents currently under investigation for their potential benefits as adjuncts to psychotherapy

\begin{tabular}{|c|c|c|c|c|}
\hline Substance & $\begin{array}{c}\text { Derivation or } \\
\text { chemical analogues }\end{array}$ & General effects and properties & Potential harms* & $\begin{array}{c}\text { Potential therapeutic } \\
\text { usest }\end{array}$ \\
\hline LSD & $\begin{array}{l}\text { Ergot fungus } \\
\text { (Claviceps purpurea); } \\
\text { morning glory } \\
\text { (Turbina corymbosa); } \\
\text { Hawaiian baby } \\
\text { woodrose (Argyreia } \\
\text { nervosa) - sources } \\
\text { of ergine or lysergic } \\
\text { acid amide }\end{array}$ & $\begin{array}{l}\text { - } 5-\mathrm{HT}_{2 \mathrm{~A}} \text { (serotonin) agonist of } \\
\text { pyramidal neurons } \\
\text { - Dizziness, weakness, tremors, } \\
\text { paresthesia } \\
\text { - Altered consciousness (visions, } \\
\text { auditory distortions, ideations) } \\
\text { - Altered mood (happy, sad, } \\
\text { fearful, irritable) } \\
\text { - Distorted sense of space, time }\end{array}$ & $\begin{array}{l}\text { - Psychosis } \\
\text { - Hallucinogen } \\
\text { persisting } \\
\text { perception } \\
\text { disorder }\end{array}$ & $\begin{array}{l}\text { - Addiction (e.g., } \\
\text { alcohol) } \\
\text { - Anxiety associated } \\
\text { with terminal illness }\end{array}$ \\
\hline Psilocybin & $\begin{array}{l}\text { Psilocybe and other } \\
\text { genera of } \\
\text { mushrooms (various } \\
\text { species) }\end{array}$ & $\begin{array}{l}\text { - } 5-\mathrm{HT}_{2 \mathrm{~A}} \text { (serotonin) agonist of } \\
\text { pyramidal neurons } \\
\text { - Dizziness, weakness, tremors, } \\
\text { paresthesia } \\
\text { - Altered consciousness (visions, } \\
\text { auditory distortions, ideations) } \\
\text { - Altered mood (happy, sad, } \\
\text { fearful, irritable) } \\
\text { - Distorted sense of space, time }\end{array}$ & $\begin{array}{l}\text { - Psychosis } \\
\text { - Hallucinogen } \\
\text { persisting } \\
\text { perception } \\
\text { disorder }\end{array}$ & $\begin{array}{l}\text { - Addiction (tobacco, } \\
\text { alcohol)6,7 } \\
\text { - Anxiety associated } \\
\text { with terminal illness }\end{array}$ \\
\hline $\begin{array}{l}\text { Ayahuasca brew } \\
\text { (admixtures contain } \\
\text { DMT) }\end{array}$ & $\begin{array}{l}\text { Chacruna leaf } \\
\text { (Psychotria viridis); } \\
\text { Chagropanga vine } \\
\text { (Diplopterys } \\
\text { cabrerana); } \\
\text { ayahuasca vine } \\
\text { (Banisteriopsis caapi); } \\
\text { assorted other } \\
\text { admixture plants }\end{array}$ & $\begin{array}{l}\text { - } 5-\mathrm{HT}_{2 \mathrm{~A}} \text { (serotonin) agonist of } \\
\text { pyramidal neurons } \\
\text { - Dizziness, weakness, tremors, } \\
\text { paresthesia } \\
\text { - Nausea, emesis } \\
\text { - Altered consciousness (visions, } \\
\text { auditory distortions, ideations) } \\
\text { - Altered mood (happy, sad, } \\
\text { fearful, irritable) } \\
\text { - Distorted sense of space, time }\end{array}$ & $\begin{array}{l}\text { - Psychosis } \\
\text { - Serotonin } \\
\text { syndrome and } \\
\text { other dangers } \\
\text { from medication } \\
\text { interactions due } \\
\text { to monoamine } \\
\text { oxidase inhibitory } \\
\text { activity }\end{array}$ & $\begin{array}{l}\text { - Addiction (alcohol, } \\
\text { cocaine, tobacco) }{ }^{9,10} \\
\text { - Depression, } \\
\text { anxiety }\end{array}$ \\
\hline Mescaline & $\begin{array}{l}\text { Peyote cactus } \\
\text { (Lophophora } \\
\text { williamsii); San Pedro } \\
\text { cactus (Echinopsis } \\
\text { pachanoi) }\end{array}$ & $\begin{array}{l}\text { - } 5-\mathrm{HT}_{2 \mathrm{~A}} \text { (serotonin) agonist of } \\
\text { pyramidal neurons } \\
\text { - Dizziness, weakness, tremors, } \\
\text { paresthesia } \\
\text { - Altered consciousness (visions, } \\
\text { auditory distortions, ideations) } \\
\text { - Altered mood (happy, sad, } \\
\text { fearful, irritable) } \\
\text { - Distorted sense of space, time }\end{array}$ & - Psychosis & - Addiction (alcohol) ${ }^{15}$ \\
\hline MDMA & $\begin{array}{l}\text { Sassafras tree } \\
\text { (Sassafras albidum) } \\
\text { - source of safrole, } \\
\text { precursor chemical }\end{array}$ & $\begin{array}{l}\text { - Serotonin, dopamine and } \\
\text { noradrenaline agonist } \\
\text { - Euphoria } \\
\text { - Arousal } \\
\text { - Perceptual alteration } \\
\text { - Enhanced empathy and } \\
\text { sociability }\end{array}$ & $\begin{array}{l}\text { - Potential } \\
\text { neurocognitive } \\
\text { deficits (e.g., } \\
\text { memory } \\
\text { impairment) } \\
\text { - Sleep disruption } \\
\text { - Short-term } \\
\text { depression }\end{array}$ & - PTSD ${ }^{16-18}$ \\
\hline \multicolumn{5}{|c|}{$\begin{array}{l}\text { Note: } \mathrm{DMT}=\text { dimethyltryptamine, LSD = lysergic acid diethylamide, MDMA = methylenedioxymethamphetamine, PTSD = posttraumatic stress disorder. } \\
\text { *Potential harms identified here are associated with illicit and unsupervised nonmedical uses of psychedelic substances (often in the context of polysubstance } \\
\text { use); current clinical studies on psychedelic agents have not reported such chronic adverse sequelae. } \\
\text { †Potential therapeutic uses are identified based on evidence from past (i.e., 1950s-1960s) and current research on psychedelic drugs. }\end{array}$} \\
\hline
\end{tabular}


trait anxiety, but significant reductions in state anxiety.

Follow-up with nine participants one year after treatment showed a sustained therapeutic benefit with no acute or chronic drug-related severe adverse events, and there were no adverse effects lasting more than one day after an LSD-assisted session. ${ }^{4}$

Psilocybin has likewise shown promise as a treatment for anxiety in patients with terminal illness. ${ }^{8}$ A 2008 study on ameliorating end-oflife anxiety focused on 12 participants with endstage cancer. ${ }^{8}$ After several non-drug-assisted therapy sessions, participants underwent a within-subject crossover study in which they received the experimental medication $(0.2 \mathrm{mg} / \mathrm{kg}$ psilocybin) and the active placebo $(250 \mathrm{mg}$ of niacin) across two sessions a few weeks apart. Findings showed that psilocybin-assisted psychotherapy lowered anxiety and improved mood, without clinically significant adverse effects. ${ }^{8}$

MDMA-assisted therapy is also being studied as a treatment for social anxiety in adults with autism, although findings have yet to be published. ${ }^{22}$

\section{Addiction}

Researchers in the 1950s and 1960s studied the use of psychedelic-assisted therapy for the treatment of addictions such as alcohol dependence, ${ }^{23}$ some key findings of which were recently reviewed in a meta-analysis that suggested a significant beneficial effect. ${ }^{3}$ In renewed clinical research on treating alcohol dependence with psilocybin-assisted therapy, a New Mexico team recruited 10 participants with a diagnosis of active alcohol dependence (and no concurrent mental illness or other substance use disorder). ${ }^{6}$ Participants received pre- and post-psychosocial support (motivational enhancement therapy) over 12 weeks, with one or two intervening open-label sessions at weeks four $(0.3 \mathrm{mg} / \mathrm{kg}$ psilocybin, $n=10)$ and eight $(0.4 \mathrm{mg} / \mathrm{kg}$ psilocybin, $n=$ 6 , or $0.3 \mathrm{mg} / \mathrm{kg}$ psilocybin, $n=1$ ). Among the participants who completed the study, the self-reported mean percent drinking days and percent heavy drinking days were reduced by more than half of what had been reported at baseline. ${ }^{6}$ Acute adverse effects such as nausea and mild headaches were reported by some participants, but no clinically significant or lasting harms resulted from the administration of psilocybin.

Other recent research on psilocybin-assisted psychotherapy for addiction includes a pilot study of treatment for tobacco dependence. This investigation was an open-label design involving 15 participants who smoked at least 10 cigarettes per day and had multiple previous unsuccessful cessation attempts. ${ }^{7}$ Participants received cognitive behavioural therapy before and after treatment with psilocybin. Treatment included two or three psilocybin-assisted psychotherapy sessions (doses of either $20 \mathrm{mg} / 70 \mathrm{~kg}$ or $30 \mathrm{mg} / 70 \mathrm{~kg}$ ), with the first session occurring on the target quit date. At six months' follow-up, 12 of the 15 participants were abstinent (biologically verified by exhaled carbon monoxide and urinary cotinine levels). ${ }^{7}$ Smoking cessation outcomes were significantly correlated with a measure of mystical experience on session days, as well as retrospective ratings of personal meaning and spiritual significance of psilocybin sessions. ${ }^{24}$ The same research team is currently designing a follow-up randomized controlled study to compare a similar psilocybin intervention with nicotine-replacement therapy.

The Amazonian folk medicine ayahuasca is a plant-based preparation with the psychoactive constituents DMT, which is chemically related to psilocybin, and harmala alkaloids, which are reversible monoamine oxidase inhibitors. An observational study of an ayahuasca-assisted intervention in a Coast Salish First Nations community in British Columbia for people $(n=12)$ seeking treatment for addictions to substances such as alcohol and cocaine showed statistically significant improvements in measures of mental health and reductions in self-reported use of these substances after six months, with no lasting adverse physical or psychological effects. ${ }^{9}$

Observational research involving members of Brazilian religious groups who regularly drink ayahuasca sacramentally has shown that, compared with a matched control group, long-term regular drinkers of ayahuasca tend to have a lower prevalence of substance use,,$^{10}$ structural brain changes that do not suggest evident pathology ${ }^{11}$ and better neuropsychological performance and psychosocial adaptation..$^{12}$ Other studies involving similar populations of long-term drinkers of ayahuasca have shown lower rates of psychoactive substance use and psychopathology. ${ }^{13,14}$

Canadian researchers are currently coordinating an international research study to investigate ayahuasca's potential as a treatment for addiction, with clinical sites in Brazil, Peru and Mexico. ${ }^{25}$

Ayahuasca differs from the other substances covered in this review, inasmuch as it is a plantbased preparation of variable composition and strength, and typically used in ceremonial contexts, which makes it more difficult for researchers to isolate the factors that may contribute to therapeutic efficacy. ${ }^{26,27}$

\section{Posttraumatic stress disorder}

In a pilot randomized controlled trial investigating MDMA-assisted psychotherapy to treat chronic treatment-resistant PTSD in the United States, outcomes from 20 participants with a mean illness 
duration of 19 years showed that the experimental treatment may improve upon the best currently available pharmacotherapies and psychotherapies. ${ }^{16}$ The clinical protocol involved several weeks of preparatory and follow-up non-drugassisted psychotherapy, during which the members of the experimental group received two MDMA-assisted sessions. No serious adverse effects were reported. Outcomes included a significant and sustained reduction in PTSD symptoms as measured by the Clinician-Administered PTSD Scale (CAPS), with $83 \%$ of participants in the experimental group (v. $25 \%$ in the placebo group) showing a reduction in symptom severity of more than $30 \%$. Furthermore, some members of the experimental group no longer met criteria for PTSD as stated in the Diagnostic and Statistical Manual of Mental Disorders, 4th Edition (DSMIV).${ }^{16}$ A long-term follow-up study involving the same participants showed that, although two patients relapsed, 74\% (14/19) of patients still showed meaningful, sustained reductions in their CAPS scores three and a half years later. ${ }^{17}$

An additional small $(n=12)$ randomized controlled trial investigating MDMA-assisted psychotherapy for PTSD was recently completed in Switzerland. ${ }^{18}$ This study compared three fulldose MDMA-assisted sessions per patient (with non-drug-assisted therapy before and after) with low-dose active placebo in a crossover design. Participants had no serious drug-related adverse events, and although reductions in CAPS scores were not statistically significant, self-assessment of PTSD symptoms as measured by the Posttraumatic Diagnostic Scale questionnaire was significantly reduced.

In 2015, researchers in Vancouver began a similar pilot study of MDMA-assisted psychotherapy for patients with PTSD, the first clinical study involving psychedelic drugs in Canada in more than 40 years.

\section{Historical lessons}

Experience from previous research - both positive and negative - has provided important lessons for current methodological designs, ethical strictures and clinical protocols and for renewed research on psychedelics involving human participants. In the 1950s and 1960s, methodological challenges confounded the advancement of psychedelic medicine, with researchers disagreeing about the suitability of randomized controlled trials and the possibility of double-blinding. ${ }^{28}$ More infamously, egregious violations of ethical protocols, such as lack of informed consent (in some cases through military or intelligence agency- supported research) resulted in substantial and long-lasting harms to some patients. ${ }^{23}$ Furthermore, unsupported claims about purported benefits of psychedelics, and sometimes explicit encouragement for non-clinical use, by some members of the research community, may have contributed to unsupervised and uncontrolled recreational use of psychedelic substances. Consequently, by the mid1970s, clinical access to and professional interest in psychedelic drugs waned, leading to a quiescence in research for several decades.

Although methodological and political challenges remain to some degree, ${ }^{27}$ recent clinical studies have shown that studies on psychedelics as therapeutic agents can conform to the rigorous scientific, ethical and safety standards expected of contemporary medical research. ${ }^{29}$ For example, patients undergo careful screening, fully informed consent is obtained and protocols are approved by ethics review boards. In addition, contemporary investigators are mindful of the checkered history of psychedelic research, and are thus cautiously reserved in reporting their findings, doing so with appropriate caveats and limitations.

\section{Potential risks and their mitigation}

Most psychedelic drugs are classified and legally scheduled as having no or very limited medical purpose, a high potential for abuse and a lack of accepted safety for use under medical supervision. ${ }^{30}$ Potential health risks of these substances include the precipitation of psychotic breaks in patients with psychotic disorders or a predisposition to these disorders. ${ }^{31}$ Thus, participation in contemporary psychedelic research typically excludes people with a personal or family history of psychosis or bipolar disorder. ${ }^{29}$

A further risk associated with psychedelic drugs is Hallucinogen Persisting Perception Disorder (HPPD), sometimes known as "flashbacks," although HPPD is more uncommon and more clinically severe than the flashbacks or visual distortions sometimes described in the days following illicit use of psychedelics. ${ }^{32,33}$ However, the incidence of adverse effects such as psychosis or HPPD in the general population is believed to be relatively low, and these effects are generally associated with the use of illicitly procured psychedelic substances, which often involves polysubstance use in uncontrolled settings without supervision. ${ }^{34}$ In light of these concerns, it is worth noting that lifetime use of classic psychedelics at the population level is associated with decreased psychological distress: ${ }^{35}$ thus, potential individual instances of harm may be overshadowed by instances in which people experience benefit or no harm. 
The most common adverse effects from the administration of psychedelics under clinical supervision are limited to the time of drug action, such as acute increases in anxiety, fear, heart rate and blood pressure. ${ }^{29}$ Without careful supervision, fearful responses could lead to dangerous behaviour (e.g., fleeing the study site). In addition, delayed-onset headache is sometimes caused by psilocybin use and possibly by other classic psychedelics. ${ }^{36}$ Although adverse effects of MDMA overlap somewhat with those of classic psychedelics, cardiovascular effects (e.g., tachycardia) are generally greater with MDMA, whereas adverse psychological reactions are more likely with classic psychedelics. It is important to note that acute adverse effects are readily managed ${ }^{37}$ and that, as described previously, none of the new clinical research studies have reported long-term harms.

The clinical protocols for contemporary psychedelic studies draw on lessons learned from the earlier era of psychedelic research, and incorporate some common elements to minimize risks and maximize potential therapeutic benefit. After obtaining fully informed consent from the patient, clinical sessions take place in health care facilities, in quiet treatment rooms with pleasant and comfortable decor. Headphones deliver music, hospital and laboratory equipment are minimal and discreetly placed, and a two-person cotherapist team is in attendance throughout the drug's action. During a session, interaction between patient and therapists is kept to a minimum, with the patient encouraged to spend much of the time engaging in self-reflection while listening to carefully selected music. Follow-up sessions that are non-drug assisted provide opportunities to integrate the insights gleaned from the experimental sessions. As research on psychedelic medicine advances, further refinements in screening, safety and therapeutic protocols will be possible.

\section{Questions for future research}

Numerous scientific and empirical questions remain in the field of psychedelic medicine. With respect to basic neuroscience research, progress in understanding the human brain and its functional relationship to mind and consciousness would be substantially advanced by further determining how psychedelic drugs work neuropharmacologically. ${ }^{30}$ This kind of knowledge would in turn be useful in applied fields such as psychology, psychiatry and addiction medicine, both to help explain mechanisms for the therapeutic results that renewed psychedelic studies are yielding and to advance understanding about optimal therapeutic protocols for these forms of treatment. With respect to clinical applications, different psychedelic medications may be indicated for different specific illnesses. Further research should elucidate not only respective efficacy, but also optimal pharmacotherapeutic and ancillary psychotherapeutic choices.

Beyond basic research on neuropharmacological mechanisms and clinical outcomes are potential economic arguments for psychedelic therapies. Substance dependence and mental disorders, such as depression and anxiety, are substantial and growing sources of illness and health system costs worldwide. ${ }^{38,39}$ Given these trends, investment of resources into researching novel treatments for mental and substance use disorders is warranted. Because preliminary evidence suggests psychedelic therapies require relatively time-limited interventions (i.e., they do not involve long-term ongoing courses of pharmacotherapeutic intervention), they may prove to be economically viable in comparison with currently available treatments.

\section{Conclusion}

Renewed scientific interest in psychedelic medicine is generating new knowledge about a class of pharmacologic substances that humans have long used for ceremonial, therapeutic and cultural purposes. As this field of research evolves, medical school curricula may need to be updated to include the latest knowledge about psychedelic drugs. This would encompass scientific evidence about relative risks and harms of psychedelic drugs which is largely absent in current drug control scheduling classifications ${ }^{40}$ and reflects adverse outcomes from uncontrolled recreational use rather than supervised clinical settings. In addition, it would encompass knowledge about the potential therapeutic uses of these agents, particularly because patients may query their physicians about research findings reported in the media. If further scientific evidence accumulates on the therapeutic value of psychedelic medicines, specialized clinical training for physicians, nurses, psychologists and other health professionals will be required to meet an increased demand for such treatments.

It behooves policy-makers to be aware of and open to new approaches to treatments emerging in the field of psychedelic medicine. This is particularly important for those concerned about the growing prevalence of mental illness, including addiction, as well as its associated human, social and economic costs. This applies not only to elected officials, but also to civil servants in health ministries and research granting agencies, where advances and innovations are translated from the clinical research laboratory into options for health care improvements that are in the public interest. Currently, international drug control scheduling classifications and popular miscon- 
ceptions about the relative risks and harms of psychedelic drugs make research involving humans difficult. However, continued medical research and scientific inquiry into psychedelic drugs may offer new ways to treat mental illness and addiction in patients who do not benefit from currently available treatments. The re-emerging paradigm of psychedelic medicine may open clinical and therapeutic doors long closed.

\section{References}

1. Chwelos N, Blewett DB, Smith CM, et al. Use of d-lysergic acid diethylamide in the treatment of alcoholism. $Q J$ Stud Alcohol 1959;20:577-90.

2. Yensen R, Di Leo FB, Rhead JC, et al. MDA-assisted psychotherapy with neurotic outpatients: A pilot study. J Nerv Ment Dis 1976;163:233-45.

3. Krebs TS, Johansen PØ. Lysergic acid diethylamide (LSD) for alcoholism: meta-analysis of randomized controlled trials. J Psychopharmacol 2012;26:994-1002.

4. Gasser P, Holstein D, Michel Y, et al. Safety and efficacy of LSD-assisted psychotherapy in subjects with anxiety associated with life-threatening diseases: a randomized active placebocontrolled phase 2 pilot study. J Nerv Ment Dis 2014;202:513-20.

5. Gasser P, Kirchner K, Passie T. LSD-assisted psychotherapy for anxiety associated with a life-threatening disease: A qualitative study of acute and sustained subjective effects. J Psychopharmacol 2015;29:57-68.

6. Bogenschutz MP, Forcehimes AA, Pommy JA, et al. Psilocybinassisted treatment for alcohol dependence: a proof-of-concept study. J Psychopharmacol 2015;29:289-99.

7. Johnson MW, Garcia-Romeu A, Cosimano MP, et al. Pilot study of the 5- $\mathrm{HT}_{2 \mathrm{~A}} \mathrm{R}$ agonist psilocybin in the treatment of tobacco addiction. J Psychopharmacol 2014;28:983-92.

8. Grob CS, Danforth AL, Chopra GS, et al. Pilot study of psilocybin treatment for anxiety in patients with advanced-stage cancer. Arch Gen Psychiatry 2011;68:71-8.

9. Thomas G, Lucas P, Capler NR, et al. Ayahuasca-assisted therapy for addiction: Results from a preliminary observational study in Canada. Curr Drug Abuse Rev 2013;6:30-42.

10. Grob CS, McKenna DJ, Callaway JC, et al. Human psychopharmacology of hoasca, a plant hallucinogen used in ritual context in Brazil. J Nerv Ment Dis 1996;184:86-94.

11. Bouso JC, Palhano-Fontes F, Rodríguez-Fornells A, et al. Longterm use of psychedelic drugs is associated with differences in brain structure and personality in humans. Eur Neuropsychopharmacol 2015;25:483-92.

12. Bouso JC, González D, Fondevila S, et al. Personality, psychopathology, life attitudes and neuropsychological performance among ritual users of ayahuasca: a longitudinal study. PLoS ONE 2012;7:e42421.

13. Fábregas JM, González D, Fondevila S, et al. Assessment of addiction severity among ritual users of ayahuasca. Drug Alcohol Depend 2010;111:257-61.

14. Halpern JH, Sherwood AR, Passie T, et al. Evidence of health and safety in American members of a religion who use a hallucinogenic sacrament. Med Sci Monit 2008;14:SR15-22.

15. Bogenschutz MP, Johnson MW. Classic hallucinogens in the treatment of addictions. Prog Neuropsychopharmacol Biol Psychiatry 2015 Mar. 14. pii: S0278-5846(15)00051-2.

16. Mithoefer MC, Wagner MT, Mithoefer AT, et al. The safety and efficacy of 3,4-methylenedioxymethamphetamine-assisted psychotherapy in subjects with chronic, treatment-resistant posttraumatic stress disorder: the first randomized controlled pilot study J Psychopharmacol 2011;25:439-52.

17. Mithoefer MC, Wagner MT, Mithoefer AT, et al. Durability of improvement in posttraumatic stress disorder symptoms and absence of harmful effects or drug dependency after 3,4-methylenedioxymethamphetamine-assisted psychotherapy: a prospective long-term follow-up study. J Psychopharmacol 2013;27:28-39.

18. Oehen P, Traber R, Widmer V, et al. A randomized, controlled pilot study of MDMA ( $\pm 3,4$-Methylenedioxymethamphetamine)assisted psychotherapy for treatment of resistant, chronic posttraumatic stress disorder (PTSD). J Psychopharmacol 2013;27: 40-52.

19. Nichols DE. Hallucinogens. Pharmacol Ther 2004;101:131-81.

20. Schultes RE, Hofmann A, Rätsch C. Plants of the gods: their sacred, healing, and hallucinogenic powers. Rochester (VT): Healing Arts Press; 2001.

21. Nichols DE. Differences between the mechanism of action of MDMA, MBDB, and the classic hallucinogens - identification of a new therapeutic class: entactogens. J Psychoactive Drugs 1986; 18:305-13.

22. Danforth AL, Struble CM, Yazar-Klosinski B, et al. MDMAassisted therapy: a new treatment model for social anxiety in autistic adults. Prog Neuropsychopharmacol Biol Psychiatry Mar. 25. pii: S0278-5846(15)00060-3

23. Dyck E. Psychedelic psychiatry: LSD from clinic to campus. Baltimore: Johns Hopkins University Press; 2008.

24. Garcia-Romeu A, Griffiths RR, Johnson MW. Psilocybinoccasioned mystical experiences in the treatment of tobacco addiction. Curr Drug Abuse Rev 2014;7:157-64.

25. Rush B. Evaluating the therapeutic potential of ayahuasca for substance use problems.. Proceedings of the Second International Psychedelic Science 2013; Apr. 18-23 Berkeley (CA): Multidisciplinary Association for Psychedelic Studies; 2013.

26. Loizaga-Velder A, Verres R. Therapeutic effects of ritual ayahuasca use in the treatment of substance dependence - qualitative results. J Psychoactive Drugs 2014;46:63-72.

27. Tupper KW, Labate BC. Ayahuasca, psychedelic studies and health sciences: the politics of knowledge and inquiry into an Amazonian plant brew. Curr Drug Abuse Rev 2014;7:71-80.

28. Oram M. Efficacy and enlightenment: LSD psychotherapy and the drug amendment of 1962. J Hist Med Allied Sci 2014;69:221-50.

29. Johnson M, Richards WA, Griffiths RR. Human hallucinogen research: guidelines for safety. J Psychopharmacol 2008;22:603-20.

30. Nutt DJ, King LA, Nichols DE. Effects of Schedule I drug laws on neuroscience research and treatment innovation. Nat Rev Neurosci 2013;14:577-85.

31. Meyerhoefer MM. Serotonergic hallucinogens. In: Johnson BA, editor. Addiction medicine: science and practice. New York: Springer; 2011: 585-602.

32. Abraham HD, Aldridge AM, Gogia P. The psychopharmacology of hallucinogens. Neuropsychopharmacology 1996;14:285-98.

33. Halpern JH, Pope HG. Hallucinogen persisting perception disorder: What do we know after 50 years? Drug Alcohol Depend 2003;69:109-19.

34. Halpern JH, Pope HG. Do hallucinogens cause residual neuropsychological toxicity? Drug Alcohol Depend 1999;53:247-56.

35. Hendricks PS, Thorne CB, Clark CB, et al. Classic psychedelic use is associated with reduced psychological distress and suicidality in the United States adult population. J Psychopharmacol 2015; 29:280-8.

36. Johnson MW, Sewell RA, Griffiths RR. Psilocybin dosedependently causes delayed, transient headaches in healthy volunteers. Drug Alcohol Depend 2012;123:132-40.

37. Doblin R, Greer G, Holland J, et al. A reconsideration and response to Parrott AC (2013) "Human psychobiology of MDMA or 'Ecstasy': An overview of 25 years of empirical research. Hum Psychopharmacol 2014;29:105-8.

38. Rehm J, Mathers C, Popova S, et al. Global burden of disease and injury and economic cost attributable to alcohol use and alcohol-use disorders. Lancet 2009;373:2223-33.

39. Whiteford HA, Rehm LDJ, Baxter AJ, et al. Global burden of disease attributable to mental and substance use disorders: findings from the Global Burden of Disease study 2010. Lancet 2013:382:1575-86.

40. Nutt D, King LA, Saulsbury W, et al. Development of a rational scale to assess the harm of drugs of potential misuse. Lancet 2007;369:1047-53.

Affiliations: School of Population and Public Health (Tupper, Wood); Department of Medicine, Faculty of Medicine (Wood), University of British Columbia, Vancouver, BC; Department of Psychiatry and Behavioral Sciences (Johnson), Johns Hopkins University School of Medicine, Baltimore, Md.; Multidisciplinary Association for Psychedelic Studies Canada (Yensen), Vancouver, BC; Orenda Institute (Yensen), Manson's Landing, BC

Contributors: Kenneth Tupper and Evan Wood conceived the idea for the article. Kenneth Tupper drafted the article. Evan Wood, Richard Yensen and Matthew Johnson analyzed and interpreted the reviewed literature, and revised the manuscript critically for important intellectual content; all of the authors have approved the final version to be published and agree to act as guarantors of the work. 